\title{
Concordant Patterns of Brain Structure in Mothers with Recurrent Depression and Their Never-Depressed Daughters
}

\author{
Lara C. Foland-Ross Negin Behzadian Joelle LeMoult lan H. Gotlib \\ Department of Psychology, Stanford University, Stanford, Calif., USA
}

\author{
Key Words \\ Depression · Risk · Concordance · Cortical thickness · Brain \\ structure
}

\begin{abstract}
Background: A growing body of research has demonstrated that having a mother with a history of major depressive disorder (MDD) is one of the strongest predictors of depression in adolescent offspring. Few studies, however, have assessed neural markers of this increased risk for depression, or examined whether risk-related anomalies in adolescents at maternal risk for depression are related to neural abnormalities in their depressed mothers. We addressed these questions by examining concordance in brain structure in two groups of participants: mothers with a history of depression and their never-depressed daughters, and never-depressed mothers and their never-depressed daughters. Method: We scanned mothers with (remitted; RMD) and without (control; CTL) a history of recurrent episodes of depression and their neverdepressed daughters, computed cortical gray matter thickness, and tested whether mothers' thickness predicted daughters' thickness. Results: Both RMD mothers and their high-risk daughters exhibited focal areas of thinner cortical gray matter compared with their CTL/low-risk counterparts.
\end{abstract}

Importantly, the extent of thickness anomalies in RMD mothers predicted analogous abnormalities in their daughters; this pattern was not present in CTL/low-risk dyads. Conclusions: We identified neuroanatomical risk factors that may underlie the intergenerational transmission of risk for MDD. Our findings suggest that there is concordance in brain structure in dyads that is affected by maternal depression, and that the location, direction, and extent of neural anomalies in high-risk offspring mirror those of their recurrent depressed mothers.

c) 2016 S. Karger AG, Basel

\section{Introduction}

Having a parent with major depressive disorder (MDD) is one of the strongest predictors of the onset of depression in adolescence and young adulthood [1,2]. Forty percent of children whose parents have a clinical mood disorder will experience an episode of depression by young adulthood [3]. Maternal depression in particular has been associated with a higher risk for emotional dysfunction in children [4], including an earlier age of onset, a more severe course of depression and greater treatment resistance [5]. Despite these alarming findings,

\section{KARGER}

E-Mail karger@karger.com

www.karger.com/dne
(C) 2016 S. Karger AG, Basel

0378-5866/16/0382-0115\$39.50/0
Lara C. Foland-Ross, $\mathrm{PhD}$

Stanford University School of Medicine

401 Quarry Road, Room 1356

Stanford, CA 94305-5795 (USA)

E-Mail lfolandross@ @tanford.edu 
we know little about neuroanatomical vulnerability factors that are transmitted from depressed parents to their high-risk children.

Identifying neural anomalies that are present in both depressed individuals and their high-risk offspring allows researchers to begin to elucidate neural factors involved in the intergenerational transmission of risk for MDD. A large body of literature is documenting that depressed adults have anomalous reductions in gray matter in higher-order cortical regions involved in the cognitive processing of emotion material, such as the anterior cingulate cortex (ACC) [6,7], dorsolateral prefrontal cortex [8], and orbitofrontal cortex [7]. Researchers have also reported depression-related decreases in gray matter of the posterior cingulate [8], an area that undergirds selfreflective thought processes [9], and the fusiform [7, 10], a region involved in the processing of negatively valenced emotional faces [11].

We do not know, however, whether these anomalies are a consequence of having been depressed, or alternatively, are abnormalities that precede the onset of depression. One way to address this question is to scan individuals who are at high risk for depression but who have not yet developed a clinically significant depressive episode. Only three such studies have examined alterations in cortical gray matter in individuals at high risk for depression. In the first study, Peterson et al. [12] found that having a depressed parent (or grandparent) was associated with increased gray matter thickness of the ACC and orbitofrontal cortex and with decreased thickness across widespread areas in the right hemisphere, including the dorsolateral cortex and ACC. Because the mean age of the participants in this sample was well above the peak age of incidence of depression in the offspring of depressed parents $[5,13,14]$, however, and because some of the participants had already experienced an episode of depression before they were scanned, it is unclear whether cortical gray matter alterations in this study are markers of depression vulnerability, markers of resilience, or scars of having been depressed. In the second study, Foland-Ross et al. [15] examined whether risk-related abnormalities in cortical thickness were present in a sample of never-disordered adolescents who were at high risk for developing major depression by virtue of having a depressed mother. They found that adolescent offspring of mothers with recurrent episodes of depression exhibited reductions in gray matter of the fusiform gyrus, a cortical area that is centrally involved in the visual processing of salient facial emotions [11], and in which abnormal function and structure has been documented in individuals at cogni- tive [16], familial [17], and genetic [18] risk for depression and in adults with a history of $\operatorname{MDD}[19,20]$. In the third study, Papmeyer et al. [17] reported cortical thickness reductions in parahippocampal and fusiform gyri of unaffected young adults at familial risk for depression. This study provided further support for a role of the fusiform and surrounding cortical gray matter in the risk for depression. Importantly, the authors found that cortical thickness reductions in the fusiform became more pronounced over time in individuals who later developed a depressive disorder.

Taken together, these findings suggest that intergenerational risk for depression is associated with abnormalities in brain morphometry, particularly in the fusiform cortex. An important gap in our understanding of the neural basis of familial risk for depression, however, involves the question of whether neural anomalies in highrisk offspring are related to those of their affectively ill parents. That is, even given replicable findings of neural abnormalities across previous studies of cortical thickness in familial risk for depression, it is not clear whether morphological anomalies found in at-risk offspring mirror those of their recurrent depressed parents, or alternatively, are independent of parental brain structure. Gaining a better understanding of whether abnormalities in brain structure in depressed parents are present in their children represents a critical research goal, as such anomalies could be novel targets for prevention efforts. To examine this question, it is necessary to scan both depressed parents and their never-depressed but high-risk children. This design not only permits an examination of whether parent-child dyads with a parental history of MDD exhibit common abnormalities in neural structure, but also offers the opportunity to examine whether the magnitude of morphological alterations in parents with a history of depression is correlated (concordant) with morphological alterations in their never-depressed offspring.

In this study we addressed the question of whether abnormalities in the brain structure of depressed parents are also present in their never-depressed but high-risk offspring by examining whether mothers with a history of depression (remitted; RMD) and their never-depressed but high-risk daughters are characterized by parallel anomalous cortical thickness relative to never-depressed mothers (CTL) and their never-depressed, low-risk daughters. We assessed cortical gray matter thickness in particular, given the evidence that cortical thickness is less influenced by individual variations in surface area than is volume [21]. We first assessed the location and magnitude of alterations in cortical gray matter thickness 
in RMD mothers compared with CTL mothers. We then examined whether daughters at high and low risk for depression also differed in brain structure in the areas that differentiated RMD from CTL mothers. Finally, for those cortical areas that differed both between RMD and CTL mothers and between low- and high-risk daughters, we examined whether thickness was concordant (correlated) within mother-daughter pairs. We hypothesized that mothers with a history of recurrent MDD would exhibit thinning in cortical gray matter across widespread areas of the cortex, including areas previously implicated in a history of depression (e.g. ACC, fusiform, and temporal cortex) relative to CTL mothers, and that these alterations would also be present in their never-depressed daughters. We hypothesized further that there would be significant intergenerational concordance in cortical gray matter structure, such that mothers' cortical thickness would be directly associated with that of their daughters, regardless of mothers' history of depression.

\section{Materials and Methods}

\section{Participants}

The study was approved by Stanford University's institutional review board. Mothers provided written informed consent and daughters provided written assent. Mothers and their daughters were recruited through advertisements posted in numerous locations (e.g. internet bulletin boards, university kiosks, supermarkets, etc.). A telephone-screening interview established that the participants were fluent in English and scan eligible. The Structured Clinical Interview for the DSM-IV (SCID) [22] was administered to the mothers to assess current and lifetime Axis I disorders. The diagnostic status of daughters was assessed using the structured Kiddie Schedule for Affective Disorders and Schizophrenia for School-Age Children - Present and Lifetime version (K-SADS-PL) [23], which interviewers administered to both the girls and their mothers (regarding the girls). To assess the current severity of symptoms of depression and anxiety, mothers completed the Beck Depression Inventory - II (BDI-II) [24] and the Beck Anxiety Inventory (BAI) [25], and daughters completed the Children's Depression Inventory - Short Form (CDI-S) [26] and the Multidimensional Anxiety Scale for Children (MASC) [27].

Dyads in the high-risk group were eligible to participate in the study if (a) the daughter did not meet criteria for any past or current Axis I disorder according to both the parent and child KSADS-PL, and (b) their mothers met DSM-IV criteria for at least two distinct episodes of MDD since the birth of their daughters but did not meet criteria for current MDD. Dyads in the healthy, lowrisk group were eligible to participate if (a) the daughter did not meet criteria for any past or current Axis I disorder based on both the parent and child K-SADS-PL, and (b) their mothers did not meet criteria for any past or current Axis I disorder. Individuals were excluded from both groups if they had experienced severe head trauma or met criteria for substance abuse within the last 6 months.

Neural Concordance and Depression Risk
Acquisition of Neuroimaging Data

All participants were scanned on a 1.5-tesla GE scanner (GE Healthcare Systems, Milwaukee, Wis., USA). Anatomic images were obtained using a T1-weighted spoiled gradient-recalled echo sequence with the following parameters: repetition time $=8.924$ $\mathrm{ms}$; echo time $=1.792 \mathrm{~ms}$; flip angle $=15^{\circ}$; an in-plane resolution of $0.859 \times 0.859$, and a slice thickness of $1.5 \mathrm{~mm}$.

\section{Statistical Analyses of Demographic and Questionnaire} Measures

For demographic, behavioral, and self-report variables, we conducted two-tailed independent samples t tests to compare demographic and clinical variables between the high- and low-risk groups.

\section{Preprocessing of Cortical Thickness Data}

We used FreeSurfer (version 5.0; http://surfer.nmr.mgh.harvard.edu) to estimate cortical thickness [28-30]. Briefly, processing streams included removing nonbrain tissue, intensity normalization, segmenting gray/white matter, and aligning each image volume to a standardized space. To estimate cortical gray matter thickness, a deformable surface algorithm was applied to segmented images to extract the pial and gray/white cortical surfaces [28]. All cortical surfaces were visually inspected by one rater (N.B.) who was blinded to group membership, and manual corrections were performed, where appropriate, following previously established procedures [31]. This additional quality control step ensured the accuracy of gray/white matter segmentation, the exclusion of scalp and other nonbrain tissue, and the inclusion of brain tissue. After spatial normalization of the data to a common space template, local cortical thickness was measured by estimating the shortest distance between the position of spatially equivalent surface points on the pial surface and the gray-white matter boundary and vice versa, and averaging these 2 values. A $20-\mathrm{mm}$ full-width at half-maximum Gaussian kernel was applied to smooth the data.

\section{History of Depression and Cortical Thickness in Mothers}

To test the hypothesis that RMD mothers have regional thinning of cortical gray matter relative to CTL mothers, we fit a general linear model at each vertex with cortical thickness as the dependent variable and diagnostic group (RMD, CTL) as the independent variable. Age and total brain volume (TBV) were included as covariates, centered to the sample mean. Because the quadratic and cubic effects of age on cortical thickness were not significant, age was retained as a linear variable in the model. We included TBV as a covariate in the statistical model because it was a significant predictor of thickness across participants, even after controlling for age. Correction for multiple comparisons in resulting statistical maps was conducted using a one-tailed threshold of $\mathrm{p}<0.05$ and the Monte-Carlo simulation toolbox provided in FreeSurfer. This approach, which is based on the methods outlined by Hagler et al. [32], estimates the probability of forming a maximum cluster of that size or larger during the simulation under the null hypothesis that results in a cluster-wise probability (CWP). Monte-Carlo simulations replace FDR (false discovery rate) and FWER (family-wise error rate) procedures that are commonly used in structural or functional paradigms to correct for multiple comparisons. CWP values are reported in the Results section. 

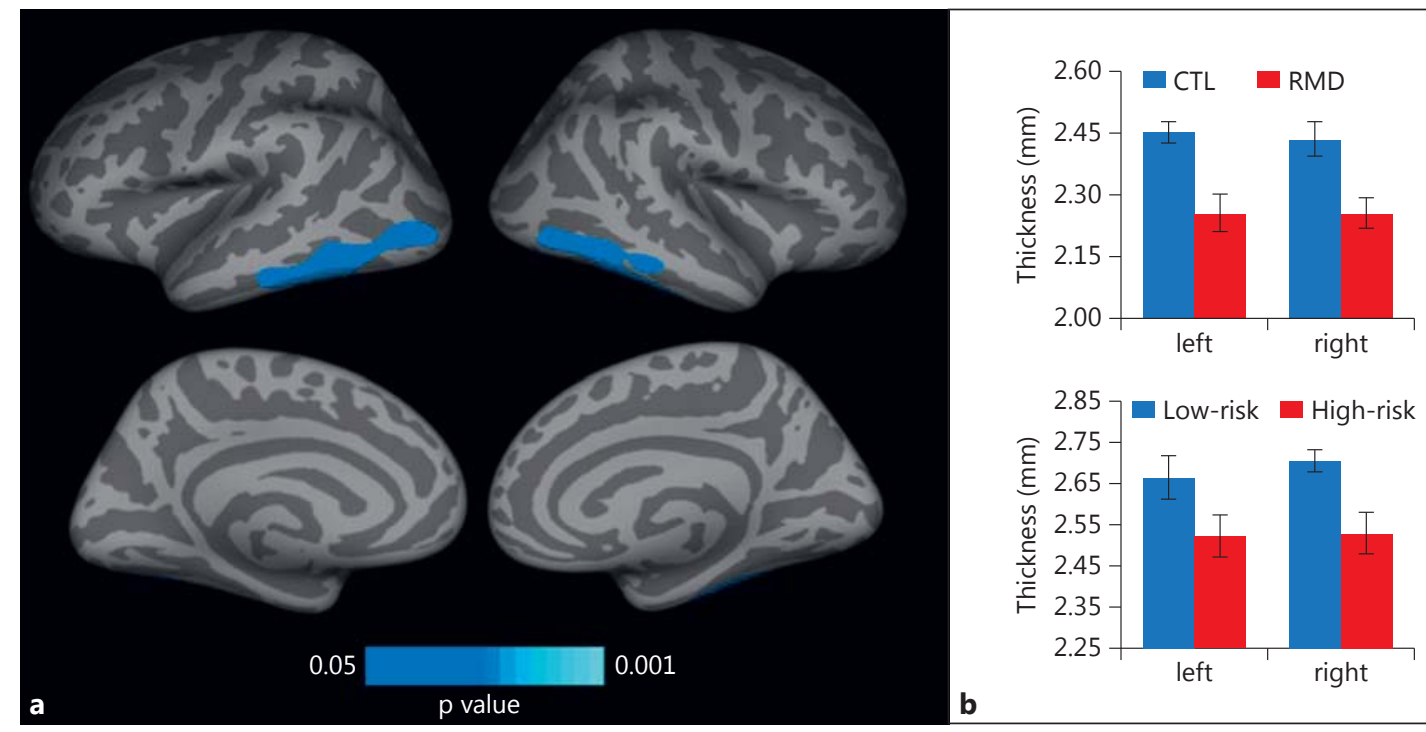

Fig. 1. a Corrected statistical significance maps showing areas of thinner cortical gray matter in recurrent depressed mothers relative to never-depressed mothers. b Mean cortical thickness from this cluster plotted for mothers (top) and daughters (bottom), for the left and right hemispheres. Plotted thickness values are adjusted for age and TBV.

\section{Maternal History of Depression and Cortical Thickness in} Daughters

To test the hypothesis that never-disordered daughters at familial risk for depression are characterized by patterns of regional gray matter thinning similar to those of their formerly depressed mothers, we conducted region of interest (ROI)-based analyses using clusters identified from corrected statistical maps in our analysis of a main effect of group in mothers. ROIs resulting from our analysis of the mothers were registered from the average template space to the cortical surface for each individual daughter using mri_label2label (part of FreeSurfer), and accurate registration was manually checked by visual inspection. We computed thickness averages separately for each ROI for every daughter. Subject-specific regional thickness averages were then adjusted for age and $\mathrm{TBV}$, and entered as the dependent variable in step 1 of a hierarchical linear regression, with group (high or low risk) entered as a predictor [33].

\section{Concordance between Mothers' and Daughters' Cortical}

Thickness

In the brain regions that showed significant main effects for both history of depression in the mothers and familial risk for depression in the daughters, we tested the hypothesis that mothers' and daughters' thickness in these ROIs were concordant. For this analysis, regional thickness averages were extracted from each mother using the ROIs described above. Raw thickness averages from these ROIs were adjusted for mothers' age and TBV and entered as a predictor of age- and TBV-adjusted daughters' regional thickness averages in step 2 of the hierarchical linear regression noted above. The interaction between the risk group and mothers' thickness was entered as a predictor in step 3.

\section{Exploratory Analyses}

While the primary aim of our study was to identify whether high- and low-risk daughters showed structural alterations in areas found to differentiate RMD from CTL mothers, we conducted additional exploratory vertex-based analyses to test whether highand low-risk daughters differed in cortical thickness in other areas for which there was a significant effect in our group analysis in the mothers (see online suppl. fig. 1S; for all online suppl. material, see www.karger.com/doi/10.1159/000444448).

\section{Results}

\section{Participant Characteristics}

Thirty-seven dyads met the study inclusion criteria. There were 14 dyads with RMD mothers and 23 dyads with CTL mothers. High- and low-risk daughters did not differ in age $\left(\mathrm{t}_{35}=-1.01, \mathrm{p}=0.32\right)$ or in scores on the CDI$\mathrm{S}\left(\mathrm{t}_{35}=-1.56, \mathrm{p}=0.13\right)$ or the MASC $\left(\mathrm{t}_{35}=0.24, \mathrm{p}=0.81\right)$. RMD and CTL mothers also did not differ in age $\left(\mathrm{t}_{35}=\right.$ $0.79, \mathrm{p}=0.44)$ or in scores on the BAI $\left(\mathrm{t}_{35}=-2.01, \mathrm{p}=\right.$ $0.06)$. RMD mothers, however, scored slightly but significantly higher on the BDI-II than did CTL mothers $\left(\mathrm{t}_{35}=\right.$ $-2.77, \mathrm{p}=0.02$; table 1 ).

Two RMD mothers met the criteria for one or more comorbid anxiety disorders (generalized anxiety disorder, $\mathrm{n}=1$; obsessive compulsive disorder, $\mathrm{n}=1$ ), and 7 were taking one or more psychotropic medications at the 


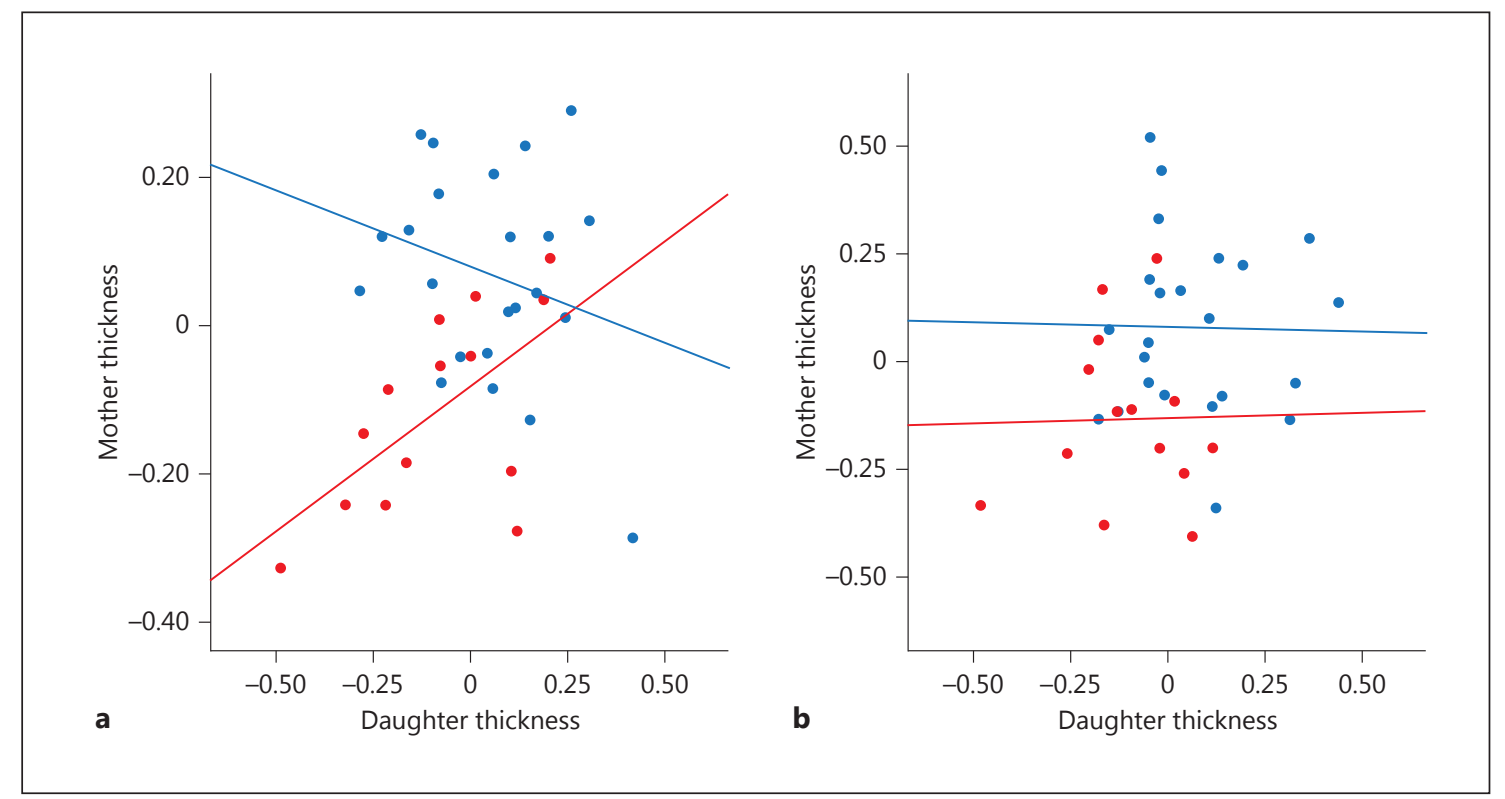

Fig. 2. Regional thickness values plotted for the left hemisphere cluster (a) and the right hemisphere cluster (b). Thickness values (in $\mathrm{mm}$ ) are adjusted for age and TBV. Blue = Dyads without a maternal history of depression; red $=$ dyads with a maternal history of depression.

time of scanning (escitalopram, $\mathrm{n}=2$; fluoxetine, $\mathrm{n}=4$; venlafaxine, $\mathrm{n}=1$; paroxetine, $\mathrm{n}=1$; citalopram, $\mathrm{n}=1$ ). Consistent with the exclusion criteria for this study, no mothers in the CTL group and no daughters in either the high- or low-risk group were taking psychotropic medications or had experienced any Axis I disorder.

\section{History of Depression and Cortical Thickness in Mothers}

Vertex-wise statistical analysis of cortical thickness maps, controlling for the linear effects of age and TBV, indicated that RMD mothers exhibited significantly thinner gray matter relative to CTL mothers in two regions: a cluster that primarily encompassed the left fusiform gyrus but also included the left inferior temporal gyrus and left lateral occipital gyrus (CWP $=0.043, \mathrm{x} / \mathrm{y} / \mathrm{z}$ Talairach peak coordinate $=-12.64 /-93.16 /-23.97)$, and a cluster that primarily encompassed the right fusiform gyrus but also included the right inferior temporal gyrus and right lateral occipital gyrus $(\mathrm{CWP}=0.025, \mathrm{x} / \mathrm{y} / \mathrm{z}$ Talairach peak coordinate $=23.85 /-69.58 /-36.10$; fig. 1a).

\section{Maternal History of Depression and Cortical \\ Thickness in Daughters}

Step 1 of the hierarchical linear regression indicated that having a mother with recurrent depression signifi-
Table 1. Participant characteristics

\begin{tabular}{lcc}
\hline a Mothers & CTL & RMD \\
\hline Age, years & $47.37 \pm 4.28$ & $46.04 \pm 5.93$ \\
Psychotropic medications, \% & 0.00 & 50.0 \\
BAI & $3.13 \pm 3.45$ & $7.71 \pm 8.12$ \\
BDI* & $2.09 \pm 2.45$ & $9.46 \pm 9.78$ \\
\hline b Daughters & Low-risk & High-risk \\
\hline Age, years & $13.29 \pm 2.41$ & $14.11 \pm 2.33$ \\
Psychotropic medications, \% & 0.00 & 0.00 \\
MASC & $38.6 \pm 14.23$ & $37.53 \pm 11.70$ \\
CDI & $0.65 \pm 1.02$ & $1.36 \pm 1.73$ \\
\hline
\end{tabular}

Data are presented as means \pm SD unless otherwise indicated. Mothers with (RMD) or without (CTL) a history of depression, and their daughters who are at high or low risk for depression based on mothers' history of the disorder. ${ }^{*} \mathrm{p}<0.05$, between groups.

cantly predicted lower thickness in daughters in both the left fusiform $\left(\beta=-0.344, \mathrm{t}_{35}=-2.169, \mathrm{p}=0.037\right.$, adjusted $\left.R^{2}=0.118\right)$ and right fusiform clusters $\left(\beta=-0.463, \mathrm{t}_{35}=\right.$ $-3.087, \mathrm{p}=0.004$, adjusted $R^{2}=0.192$; fig. $1 \mathrm{~b}$ ). Results of exploratory vertex-based analyses of a main effect of ma- 
ternal history of depression indicated that the location, magnitude, and extent of difference in cortical thickness between high- and low-risk daughters were similar to those identified in our analyses of RMD and CTL mothers. High-risk daughters did not exhibit reductions in cortical thickness relative to low-risk daughters in any brain region outside of the two clusters identified in our analysis of RMD versus CTL mothers. Details concerning the results of this analysis are presented in the online supplementary material.

\section{Concordance between Mothers' and Daughters' Cortical Thickness}

Step 2 of our hierarchical linear regression model indicated that mothers' cortical thickness did not predict daughters' cortical thickness as a main effect in either the left $\left(\beta=0.809, \mathrm{t}_{34}=0.460, \mathrm{p}=0.648\right.$, adjusted $R^{2}$ change $=0.005)$ or the right $\left(\beta=-0.005, \mathrm{t}_{34}=-0.028, \mathrm{p}=\right.$ $0.978, R^{2}$ change $<0.001$ ) fusiform cluster. In step 3 , however, the effect of mothers' cortical thickness on daughters' cortical thickness differed by group in the left fusiform cluster $\left(\beta=0.626, \mathrm{t}_{33}=2.825, \mathrm{p}=0.008, R^{2}\right.$ change $=0.171$; fig. 2 ). Tests of simple slopes indicated that whereas decreased thickness in RMD mothers' left fusiform cluster predicted analogous decreased thickness in their daughters $(\beta=0.605, p=0.022)$, cortical thickness in CTL mothers was not a significant predictor of thickness in their daughters $(\beta=-0.261, p=0.228)$. For the right fusiform cluster, the interaction between group and mothers' thickness was not significant $\left(\beta=0.022, \mathrm{t}_{33}=\right.$ $0.102, \mathrm{p}=0.920$ ).

\section{Discussion}

This is the first study to examine brain structure in both formerly depressed mothers and their never-depressed adolescent daughters who are at risk for depression by virtue of their mothers' history of the disorder. We documented two major findings in this study. First, both recurrent depressed mothers and their high-risk daughters exhibited bilateral reductions in gray matter thickness of clusters that encompassed the fusiform and inferior temporal and lateral occipital gyri relative to their low-risk counterparts. Second, concordance analyses indicated that mothers' thickness in this region in the left hemisphere directly predicted thickness in their neverdepressed daughters, a pattern that was present only in dyads in which the mother experienced clinically significant depression during the daughter's lifetime.
Our findings of reduced gray matter in the bilateral fusiform and inferior temporal and lateral occipital gyri in women with a history of depression adds to previous reports of inferotemporal cortical reductions in MDD $[20,34,35]$. The reduction in cortical gray matter that we documented in this same set of regions in high-risk daughters is also consistent with findings of investigations that have examined the neural anomalies associated with a genetic [18] or cognitive risk $[16,36]$ for depression. For example, decreased fusiform gray matter has been reported in a study of individuals who were classified as vulnerable to developing depression based on having a negative cognitive style [16]. Anomalous responding in this brain area has also been associated with high scores on a measure of neuroticism [36], a trait that is posited to predispose individuals to experience depression [37]. Finally, reduced gray matter of the fusiform was reported in a sample of individuals who were at genetic risk for depression, defined by an exchange of amino acids from valine to methionine at a single site on the brainderived neurotrophic gene [18]. The current study provides further support for a role of inferotemporal and fusiform cortex in neural risk for depression. We also extend previous findings by documenting that anomalies in the structure of this area appear to be passed down from recurrent depressed mothers to their high-risk offspring.

It is well established that the inferior temporal, fusiform, and lateral occipital cortices coactivate during the processing of faces $[11,38-40]$ and, in particular, during the processing of negative facial emotions $[11,40]$. This specialization is posited to occur in part as a result of extensive structural connections with the amygdala [41]. Functional neuroimaging studies utilizing tasks that involve the perception of emotional faces have demonstrated a dynamic interplay among these areas, whereby the occipital cortex influences both temporal and fusiform gray matter, and the fusiform, in turn, exerts a strong causal influence on activation in the amygdala [40]. Given the specialization of these regions and their involvement in the processing of salient material [42], an important direction for future research involves examining the relation between structural alterations in these regions and mood-congruent biases in cognition. Indeed, although it is not clear precisely how structural or functional alterations of this brain area might contribute to a diagnosis of or vulnerability for depression, it is tempting to speculate that anomalies in the structure and/or function of this region underlie the enhanced processing of emotionally salient information in high-risk individuals, and that these behavioral anomalies, in turn, contribute to increased 
vulnerability for the disorder. Partial support for this possibility comes from data demonstrating that biases in the processing of negatively valenced faces are present in both remitted depressed adults [43] and youth at familial risk for depression [44]. Whether neural anomalies mediate variations in the processing of negatively valenced information, however, is not yet clear.

This is the first neuroimaging study to examine parent-child concordance in brain structure. Our findings concerning concordance in the left inferotemporal gray matter is important in demonstrating, for the first time, that anomalous brain structure in this region in mothers with a history of depression is a significant determinant of aberrant brain structure in this same area in their never-disordered, but high-risk daughters. These findings increase our understanding of the neurobiological factors that underlie intergenerational risk for depression, and suggest a number of directions for future research. First, it is important that our findings be replicated in a larger sample. Moreover, following this sample longitudinally would allow us to examine whether higher concordance in mother-daughter thickness predicts a greater likelihood that the daughter will develop depression. Second, future studies should include cognitive assessments to examine behavioral aspects of structural neural alterations in remitted depressed mothers and their daughters. Finally, future studies should quantify the relative contributions of genetic and environmental factors in order to better understand the mechanisms that underlie intergenerational concordance in brain structure.

Our findings concerning concordance add to a body of research documenting parent-child concordance in other measures of psychopathology. For example, maternal levels of cortisol have been found to be among the strongest predictors of children's cortisol levels [45]. LeMoult et al. [46] recently examined parent-child concordance of this variable and found that anomalous increases in cortisol production in formerly depressed mothers predicted increased cortisol production in their high-risk daughters. This finding, taken together with research linking elevated cortisol with the subsequent onset of $\operatorname{MDD}[47,48]$, suggests that maternal neurobiological markers are important predictors of mental health outcomes in children. Future research should assess how distinct neurobiological markers (e.g. cortical thickness and cortisol) may be related to each other and how they may, independently or combined, predict increased risk for depression.

Contrary to our hypothesis, we did not observe concordance of neural measures in mother-daughter dyads who were not affected by MDD. Although the reasons for this are unclear, it is possible that the factors underlying the intergenerational concordance of temporal and occipital cortical gray matter are unique to a familial history of depression. While we cannot conclude what these factors may be, research examining the etiology of MDD clearly implicates both genetic and environmental components. For example, one study of monozygotic twins found that the fusiform is principally dependent on unique individual experience [49], whereas others have found evidence suggesting that face recognition areas of the brain are under genetic control [50]. Longitudinal studies that more clearly point to the factors that influence the development of cortical regions implicated in the processing of moodcongruent valenced material are needed.

We should note five limitations of the present study. First and foremost, the sample size was relatively small, which may have limited our power to detect group differences in other brain structures. Second, prior research indicates that a surge of newly emergent cases of MDD occurs between 15 and 18 years of age in females [51]. Because the maximum age of daughters in our sample was 20 years, we cannot rule out the possibility that some of the daughters in our study were particularly resilient to the development of depression. Third, half of the RMD mothers were receiving medication or were comorbid for one or more anxiety disorders at the time of scanning, and the effects of medication on our findings is unknown. Fourth, because we focused on cortical thickness in our study, it remains for future research to examine whether individuals with a history of depression and their offspring who are at risk for depression show convergent patterns of structural abnormality in subcortical gray matter or white matter. Finally, because none of the offspring in our study had a history of depression, it is not clear whether a different pattern of parent-child concordance would characterize dyads in which both the mother and the daughter have experienced an episode of depression. Future studies that examine whether parentchild concordance in inferotemporal gray matter varies as a function of parent-child concordance in depression history would be informative in addressing this issue.

In conclusion, the current study supports the formulation that the intergenerational transmission of anomalous structure in the fusiform, inferior temporal, and lateral occipital gyri - regions that are important to the processing of mood-congruent valenced material - is implicated in familial risk for major depression. Future studies should test whether concordance of neural anomalies in recurrent depressed mothers and their high-risk daughters is itself a significant predictor of the onset of depression in 
the daughters. In addition, investigations that elucidate both the adverse and the protective factors that influence parent-child concordance in neural structure will be critical in increasing our understanding of factors that contribute to the onset of this severe and debilitating disorder.
Schizophrenia and Affective Disorders (NARSAD; Distinguished Investigator Award to I.H.G., Young Investigator Award 19018 to L.C.F.-R., and Young Investigator Award 22337 to J.L.) and the Hope for Depression Research Foundation (to I.H.G. and L.C.F.R.). L.C.F.-R. had full access to all of the data in the study and takes responsibility for the integrity of the data and the accuracy of the data analysis.

\section{Acknowledgments}

This work was supported by grants from the National Institute of Mental Health (MH74849 to I.H.G., MH090617 to L.C.F.-R., and MH102013 to J.L.), the National Alliance for Research in

\section{Disclosure Statement}

All authors report no competing or financial interests.

\section{References}

$\checkmark 1$ Beardslee WR, Versage EM, Gladstone TR: Children of affectively ill parents: a review of the past 10 years. J Am Acad Child Adolesc Psychiatry 1998;37:1134-1141.

- 2 Williamson DE, Birmaher B, Axelson DA, Ryan ND, Dahl RE: First episode of depression in children at low and high familial risk for depression. J Am Acad Child Adolesc Psychiatry 2004;43:291-297.

3 Goodwin F: Depression and Manic Depressive Illness. Bethesda, National Institutes of Health, 1982.

4 Connell AM, Goodman SH: The association between psychopathology in fathers versus mothers and children's internalizing and externalizing behavior problems: a meta-analysis. Psychol Bull 2002;128:746-773.

5 Lieb R, Isensee B, Höfler M, Pfister H, Wittchen $\mathrm{H}-\mathrm{U}$ : Parental major depression and the risk of depression and other mental disorders in offspring: a prospective-longitudinal community study. Arch Gen Psychiatry 2002;59: 365-374.

6 Bora E, Fornito A, Pantelis C, Yücel M: Gray matter abnormalities in major depressive disorder: a meta-analysis of voxel based morphometry studies. J Affect Disord 2012;138: 9-18.

7 Koolschijn PCMP, van Haren NEM, Lensvelt-Mulders GJLM, Hulshoff Pol HE, Kahn RS: Brain volume abnormalities in major depressive disorder: a meta-analysis of magnetic resonance imaging studies. Hum Brain Mapp 2009;30:3719-3735.

8 Grieve SM, Korgaonkar MS, Koslow SH, Gordon E, Williams LM: Widespread reductions in gray matter volume in depression. Neuroimage Clin 2013;3:332-339.

-9 Johnson MK, Raye CL, Mitchell KJ, Touryan SR, Greene EJ, Nolen-Hoeksema S: Dissociating medial frontal and posterior cingulate activity during self-reflection. Soc Cogn Affect Neurosci 2006;1:56-64.
10 Gotlib IH, Hamilton JP: Neuroimaging and depression: current status and unresolved issues. Curr Dir Psychol Sci 2008;17:159-163.

-11 Vuilleumier P, Armony JL, Driver J, Dolan RJ: Effects of attention and emotion on face processing in the human brain: an event-related fMRI study. Neuron 2001;30:829-841.

12 Peterson B, Warner V, Bansal R, et al: Cortical thinning in persons at increased familial risk for major depression. Proc Natl Acad Sci USA 2009;106:6273-6278.

13 Hammen C, Brennan PA, Shih JH: Family discord and stress predictors of depression and other disorders in adolescent children of depressed and nondepressed women. J Am Acad Child Adolesc Psychiatry 2004;43:9941002.

14 Weissman MM, Gammon GD, John K, et al: Children of depressed parents: increased psychopathology and early onset of major depression. Arch Gen Psychiatry 1987;44:847853.

15 Foland-Ross LC, Gilbert B, Joormann J, Gotlib IH: Neural markers of familial risk for depression: an investigation of cortical thickness abnormalities in healthy adolescent daughters of mothers with recurrent depression. J Abnorm Psychol 2015;124:476-485.

16 Zhang X, Yao S, Zhu X, Wang X, Zhu X, Zhong M: Gray matter volume abnormalities in individuals with cognitive vulnerability to depression: a voxel-based morphometry study. J Affect Disord 2012;136:443-452.

17 Papmeyer M, Giles S, Sussmann JE, et al: Cortical thickness in individuals at high familial risk of mood disorders as they develop major depressive disorder. Biol Psychiatry 2015;78: 58-66.

18 Montag C, Weber B, Fliessbach K, Elger C, Reuter M: The BDNF Val66Met polymorphism impacts parahippocampal and amygdala volume in healthy humans: incremental support for a genetic risk factor for depression. Psychol Med 2009;39:1831-1839.
19 Stuhrmann A, Suslow T, Dannlowski U: Facial emotion processing in major depression: a systematic review of neuroimaging findings. Biol Mood Anxiety Disord 2011;1:10.

20 Lee H-Y, Tae WS, Yoon H-K, et al: Demonstration of decreased gray matter concentration in the midbrain encompassing the dorsal raphe nucleus and the limbic subcortical regions in major depressive disorder: an optimized voxel-based morphometry study. J Affect Disord 2011;133:128-136.

21 Winkler AM, Kochunov P, Blangero J, et al: Cortical thickness or grey matter volume? The importance of selecting the phenotype for imaging genetics studies. Neuroimage 2010;53:1135-1146.

22 First M, Spitzer R, Gibbon M, Williams J: Structured Clinical Interview for DSM-IV Axis I Disorders - Clinician Version (SCIDCV). Washington, American Psychiatric Press, 1996.

23 Kaufman J, Birmaher B, Brent D, et al: Schedule for Affective Disorders and Schizophrenia for School-Age Children - Present and Lifetime Version (K-SADS-PL): initial reliability and validity data. J Am Acad Child Adolesc Psychiatry 1997;36:980-988.

24 Beck A, Ward C, Mendelson M, Mock J, Erbaugh J: An inventory for measuring depression. Arch Gen Psychiatry 1961;4:561-571.

25 Beck AT, Epstein N, Brown G, Steer RA: An inventory for measuring clinical anxiety: psychometric properties. J Consult Clin Psychol 1988;56:893-897.

26 Kovacs, M: Children's Depression Inventory, ed 2. New York, Multi-Health Systems, 1992.

-27 March JS, Parker JDA, Sullivan K, Stallings P, Conners CK: The Multidimensional Anxiety Scale for Children (MASC): factor structure, reliability, and validity. J Am Acad Child Adolesc Psychiatry 1997;36:554-565. 
28 Dale AM, Fischl B, Sereno MI: Cortical surface-based analysis. I. Segmentation and surface reconstruction. Neuroimage 1999;9:179194.

29 Fischl B, Dale AM: Measuring the thickness of the human cerebral cortex from magnetic resonance images. Proc Natl Acad Sci USA 2000; 97:11050-11055.

-30 Salat D, Buckner R, Snyder A, et al: Thinning of the cerebral cortex in aging. Cereb Cortex 2004; 14:721-730.

- 31 Black JM, Tanaka H, Stanley L, et al: Maternal history of reading difficulty is associated with reduced language-related gray matter in beginning readers. Neuroimage 2012;59:30213032.

32 Hagler DJ, Saygin AP, Sereno MI: Smoothing and cluster thresholding for cortical surfacebased group analysis of fMRI data. Neuroimage 2006;33:1093-1103.

33 Cohen J, Cohen P, West SG, Aiken LS: Applied Multiple Regression/Correlation Analysis for the Behavioral Sciences. Mahwah, Erlbaum, 2003.

34 Alemany S, Mas A, Goldberg X, et al: Regional gray matter reductions are associated with genetic liability for anxiety and depression: an MRI twin study. J Affect Disord 2013;149: 175-181.

35 Leung K-K, Lee TMC, Wong MMC, Li LSW, Yip PSF, Khong P-L: Neural correlates of attention biases of people with major depressive disorder: a voxel-based morphometric study. Psychol Med 2009;39:1097-1106.
36 Chan SWY, Norbury R, Goodwin GM, Harmer CJ: Risk for depression and neural responses to fearful facial expressions of emotion. Br J Psychiatry J Ment Sci 2009;194:139145.

37 Roberts JE, Gotlib IH: Lifetime episodes of dysphoria: gender, early childhood loss and personality. Br J Clin Psychol Br Psychol Soc 1997;36:195-208.

38 Haxby JV, Hoffman EA, Gobbini MI: The distributed human neural system for face perception. Trends Cogn Sci 2000;4:223-233.

39 Kanwisher N, McDermott J, Chun MM: The fusiform face area: a module in human extrastriate cortex specialized for face perception. J Neurosci 1997;17:4302-4311.

40 Fairhall SL, Ishai A: Effective connectivity within the distributed cortical network for face perception. Cereb Cortex 2007;17:24002406.

41 Amaral D, Price J, Pitakanen A, Carmichael S: Anatomical Organization of the Primate Amygdaloid Complex. New York, WileyLiss, 1992.

42 Vuilleumier P, Schwartz S: Emotional facial expressions capture attention. Neurology 2001;56:153-158

43 Joormann J, Gotlib IH: Selective attention to emotional faces following recovery from depression. J Abnorm Psychol 2007;116:80-85.

44 Joormann J, Talbot L, Gotlib IH: Biased processing of emotional information in girls at risk for depression. J Abnorm Psychol 2007; 116:135-143.
5 Bright MA, Granger DA, Frick JE: Do infants show a cortisol awakening response? Dev Psychobiol 2012;54:736-743.

46 LeMoult J, Chen MC, Foland-Ross LC, Burley $\mathrm{H}$, Gotlib IH: Concordance of mother-daughter diurnal cortisol production: understanding the intergenerational transmission of risk for depression. Biol Psychol 2015;108:98104.

47 Halligan S, Herbert J, Goodyer I, Murray L: Disturbances in morning cortisol secretion in association with maternal postnatal depression predict subsequent depressive symptomatology in adolescents. Biol Psychiatry 2007; 62:40-46.

48 Adam EK, Doane LD, Zinbarg RE, Mineka S, Craske MG, Griffith JW: Prospective prediction of major depressive disorder from cortisol awakening responses in adolescence. Psychoneuroendocrinology 2010;35:921-931.

49 Pinel P, Lalanne C, Bourgeron T, et al: Genetic and environmental influences on the visual word form and fusiform face areas. Cereb Cortex 2015;25:2478-2493.

50 Polk TA, Park J, Smith MR, Park DC: Nature versus nurture in ventral visual cortex: a functional magnetic resonance imaging study of twins. J Neurosci 2007;27:13921-13925.

51 Hankin BL, Abramson LY, Moffitt TE, Silva PA, McGee R, Angell KE: Development of depression from preadolescence to young adulthood: emerging gender differences in a 10 year longitudinal study. J Abnorm Psychol 1998;107:128-140. 